Check for updates

The BMJ

Cite this as: BMJ2020;371:m4267 http://dx.doi.org/10.1136/bmj.m4267 Published: 03 November 2020

\section{Covid-19: Study findings strongly support use of pooled testing, say researchers}

Gareth lacobucci

Pooled testing could be an efficient and effective way of processing large numbers of covid-19 tests, a preprint study ${ }^{1}$ has reported.

Pooling has been proposed as a strategy to reduce costs and increase throughput of tests. The process-which involves mixing several samples together, testing the pooled sample, and testing people in the group individually if the result comes back positive-has already been deployed at some UK universities to help stop outbreaks and keep campuses open. ${ }^{2}$

To assess the efficiency and sensitivity of pooled testing, researchers tested 133816 swab samples collected between April and September 2020. Samples from symptomatic and hospitalised patients were tested individually for SARS-CoV-2, while samples from screened asymptomatic individuals, such as hospital staff and nursing homes residents, were pooled.

Their findings, published on medRxiv, show that 32466 reverse transcription polymerase chain reaction (RT-PCR) tests were used, meaning the pooled approach saved 101350 (76\%) RT-PCR reactions compared with individual testing. The team reported a clinically insignificant sensitivity loss of three cycle thresholds, and a false positive rate between $3.9 \%$ and $5.3 \%$.

"We were able to spare $76 \%$ of RNA extraction and RT-PCR tests, even in the reality of frequently changing prevalence rate $(<1 \%$ to $6 \%)$," the authors said. When the prevalence rate in the pooled samples increased, the researchers switched from pooling in groups of eight to pooling in groups of five. They alternated the pool size between eight and five thereafter to maintain optimal pooling efficiency.

They calculated that the empirical efficiency of pooled testing (defined as the total number of tested samples divided by the total number of actual RT-PCR reactions performed) was 4.587 and 2.377 for the eight and five sample pools, respectively. They noted that these values were better than the expected optimal efficiency values for both the eight and the five pool sizes, (3.955 and 2.1891, respectively). "Surprisingly, we observed pooling efficiency and sensitivity that exceed theoretical predictions, which resulted from non-random distribution of positive samples in pools," the authors said. "Overall, the findings strongly support the use of pooling for efficient large high throughput SARS-CoV-2 testing.”

The authors suggested that while increased prevalence rates should theoretically lead to decreased efficiency if samples arrive at random to diagnostic laboratories, this was less of an problem if, in reality, samples arrived in batches from places such as colleges, nursing homes, or hospitals. "We sort samples into pools as they arrive at the lab, such that family members and roommates are often pooled together aiming to increase the number of positive samples within the pool," they said.

"Our empirical evidence from testing over 130,000 samples in pools strongly projects on the feasibility and benefits of carefully conducted pooling for surveillance, control, and community re-openings,” they added.

Barak N, Ben-Ami R, Sido T, et al. Lessons from applied large-scale pooling of 133816 SARS-CoV-2 RT-PCR tests.MedRxiv 2020.10.16.20213405 [Preprint] 2020. doi: 10.1101/2020.10.16.20213405.

2 Mahase E. Covid-19: Universities roll out pooled testing of students in bid to keep campuses open. BMJ 2020;370:m3789. doi: 10.1136/bmj.m3789 pmid: 32994203 